\title{
Viscous Behavior of Binary Mixtures of Mesogenic Solvent/Non-Mesogenic Solute
}

\author{
J. JADŻYn, G. CzEChowski AND S. LAskowski \\ Institute of Molecular Physics, Polish Academy of Sciences \\ M. Smoluchowskiego 17, 60-179 Poznań, Poland
}

(Received March 3, 2006)

\begin{abstract}
The paper presents results of the shear viscosity measurements performed on diluted binary mixtures of mesogenic solvent $n$-hexylcyanobiphenyl $\left(\mathrm{C}_{6} \mathrm{H}_{13} \mathrm{PhPhC} \equiv \mathrm{N}, 6 \mathrm{CB}\right)$ and two non-mesogenic admixtures: (i) $n$-heptylcyanophenyl $\left(\mathrm{C}_{7} \mathrm{H}_{15} \mathrm{PhC} \equiv \mathrm{N}, 7 \mathrm{CP}\right)$, composed of the molecules of the same polarity as the solvent molecules but of a slightly shorter length, and (ii) 4- $n$-propylcyclohexyl-4'- $n$-pentylphenyl $\left(\mathrm{C}_{3} \mathrm{H}_{7} \mathrm{CyHxPhC} \mathrm{H}_{11}, 3 \mathrm{CyP} 5\right)$, composed of the non-polar molecules but of a length very close to that of the mesomorphic solvent molecules. The experiment showed that the concentrational depression of the clearing temperature and the temperature extent of the isotropic + nematic $(\mathrm{I}+\mathrm{N})$ two-phase region in the mixtures are significantly smaller, i.e. the nematic phase is more thermodynamically stable, when the admixture molecular length is compatible to that of the mesogenic solvent, regardless of the polarity of the admixture molecules. The activation energy for freely flowing mixtures in the isotropic, nematic, and two-phase $\mathrm{I}+\mathrm{N}$ regions was determined and discussed.
\end{abstract}

PACS numbers: 64.70.Md, 66.20.+d, 83.80.Xz, 83.85.Jn

\section{Introduction}

Phase transitions are classified as the first or the second order, depending on the behavior of the first and second derivatives of the thermodynamic potential relevant to the system under investigation [1-3]. In the cases of liquids or liquid crystals, where the experiments are performed mainly in the isothermal or isobaric conditions, a behavior of the Gibbs function $G(T, p)$ derivatives: $(\partial G / \partial p)_{T}=V,-(\partial G / \partial T)_{p}=S,\left(\partial^{2} G / \partial T^{2}\right)_{p}=C_{p} / T,\left(\partial^{2} G / \partial p^{2}\right)_{T}=V K$, and $\partial^{2} G / \partial p \partial T=V \alpha$, is the basis of the classification. The first order phase transitions are accompanied by a jump in the specific volume, $\Delta V$, and the entropy, $\Delta S$. At a second order transition these quantities are continuous but jumps in the heat capacity at a constant pressure $\left(\Delta C_{p}\right)$, the isothermal compressibility $(\Delta K)$, and the expansion coefficient $(\Delta \alpha)$, are observed $[4,5]$. 
As the entropy jump is related to the latent heat: $\Delta H=T_{\mathrm{tr}} \Delta S$, where $T_{\mathrm{tr}}$ is the temperature of transition, calorimetric measurements are the main tool for determination of the phase transition order. At a typical first order transition, namely in the melting from a crystalline solid to isotropic liquid or to liquid crystalline phase, the enthalpy change is around $20-50 \mathrm{~kJ} / \mathrm{mol}$, so, from the experimental point of view, there are no problems with detection of such heating effects. The problem arises for transitions which are accompanied by a small or very small latent heat. This is the case for transitions from one liquid crystal phase to other liquid crystal phase or from a liquid crystal phase to isotropic liquid, where the enthalpy changes are of the order of $1 \mathrm{~kJ} / \mathrm{mol}$, and often much less. Such types of phase transitions are called a weakly first order or close to the second order and for their detection scanning calorimeters of a high resolution have to be used $[5,6]$.

The existence of the entropy jump at a first order transition in a given pure compound has consequences in mixtures of the compound with a solute - the two-phase region appears. So, the entropy jump observed at the transition from the liquid crystalline nematic phase to the isotropic liquid, which, as it was mentioned above, usually has a relatively small value, leads to an appearance of the nematic + isotropic two-phase region in a mixture of nematogenic compound with the non-mesogenic admixture. In this paper we present the experimental data on the phase diagrams of diluted binary mixtures of mesogenic solvent and two non-mesogenic admixtures of different structure and polarity. The results of measurements of the viscous properties of the freely flowing mixtures in the nematic, isotropic phases, and two-phase nematic + isotropic regions are presented.

\section{Experimental}

$n$-hexylcyanobiphenyl $\left(\mathrm{C}_{6} \mathrm{H}_{13} \mathrm{PhPhCN}, 6 \mathrm{CB}, T_{\mathrm{NI}}=29.8^{\circ} \mathrm{C}\right), n$-heptylcyanophenyl $\left(\mathrm{C}_{7} \mathrm{H}_{15} \mathrm{PhCN}, 7 \mathrm{CP}\right)$, and 4- $n$-propylcyclohexyl-4'- $n$-pentylphenyl $\left(\mathrm{C}_{3} \mathrm{H}_{7} \mathrm{CyHxPhC}_{5} \mathrm{H}_{11}, 3 \mathrm{CyP} 5\right)$ were synthesized and purified at the Institute of Chemistry, Military University of Technology, Warsaw. The purity of the compounds, checked by chromatography, was better than $99 \%$. The temperature of the phase transitions in pure $6 \mathrm{CB}$ and in the solutions were determined using the polarizing microscope.

The viscosity was measured with a Haake viscometer RV 20 with the measuring system CV 100. The system consists of a rotary beaker filled with a studied compound and a cylindrical sensor of Mooney-Ewart type (M 15) placed in the center of the beaker. The liquid gap was $0.5 \mathrm{~mm}$. The accuracy of the viscosity measurements was $0.5 \%$. The temperature of the sample was controlled with an accuracy of $0.1^{\circ} \mathrm{C}$.

\section{Results and discussion}

Figure 1 presents the phase diagrams of the binary mixtures of $6 \mathrm{CB}$ and two non-mesogenic liquids. The first one $(7 \mathrm{CP})$ is composed of the molecules of 


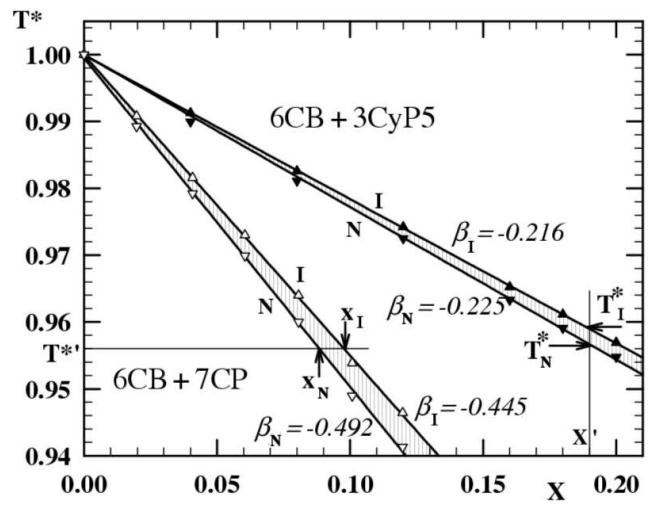

Fig. 1. Phase diagrams for mixtures of mesogenic $6 \mathrm{CB}$ and non-mesogenic $7 \mathrm{CP}$ and 3CyP5. $T^{*}=T / T_{\mathrm{NI}}$ is the reduced temperature and $x$ is the mole fraction of non-mesogenic solute. The values of the slope $\beta$ of the diagram lines are shown. The hatched area corresponds to the two-phase I $+\mathrm{N}$ region.

the same polarity as $6 \mathrm{CB}$ but of the length $\left(l_{7 \mathrm{CP}}=15.2 \AA\right)$ not consistent with $6 \mathrm{CB}$ molecules $\left(l_{6 \mathrm{CB}}=18.3 \AA\right)$. The second one $(3 \mathrm{CyP} 5)$ is a non-polar liquid composed of the molecules of the length $\left(l_{3 \mathrm{CyP} 5}=18.4 \AA\right)$ very close to that of the $6 \mathrm{CB}$ molecules. The difference in the slopes of the diagram lines representing the depression of temperatures of the phase transitions in these two mixtures is important.

The extent of the two-phase $\mathrm{I}+\mathrm{N}$ region can be represented in two ways: as a temperature extent $\left(\Delta T_{\mathrm{I}+\mathrm{N}}\right)$ at a constant concentration of admixture or as a concentration of admixture extent $\left(x_{\mathrm{I}}-x_{\mathrm{N}}\right)$ at a constant temperature, as depicted in Fig. 1. Both representations are important and interesting as they give a different look at the isotropic to the nematic phase transition. The temperature extent of the two-phase region can be observed directly with the polarizing microscope and, as presented in Fig. 2, gives an answer on the role of the molecular parameters of admixture on the nematic phase stability in the mixture. Our results show that the nematic phase is more thermodynamically stable when the molecular length of admixture is compatible to that of the mesogenic solvent, and the polarity of the admixture molecules plays a secondary role. The effect was previously observed in some other experiments [7].

The importance of the concentration extent of the isotropic + nematic two-phase region results from the statistical theory of binary mixtures of mesogenic + non-mesogenic compounds proposed by Martire et al. [8, 9]. According to the theory, the extent of the two-phase region $\left(x_{\mathrm{I}}-x_{\mathrm{N}}\right)$, as a function of the reduced temperature $T^{*}\left(=T / T_{\mathrm{NI}}\right)$, does not depend on the solute used but only on the magnitude of the entropy jump $\Delta S_{\mathrm{NI}}$ in pure mesogenic solvent:

$$
x_{\mathrm{I}}-x_{\mathrm{N}}=\frac{\Delta S_{\mathrm{NI}}}{R}\left(1-T^{*}\right),
$$




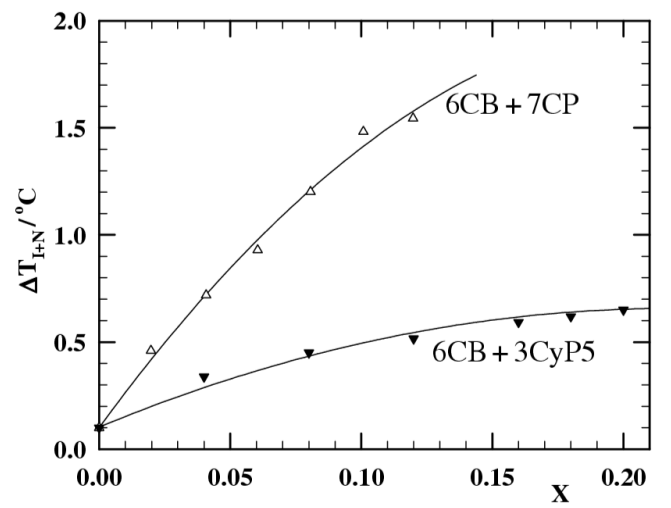

Fig. 2. Temperature extent of the two-phase $\mathrm{I}+\mathrm{N}$ region in $6 \mathrm{CB}+$ non-mesogenic solute mixtures as a function of the mole fraction of the solutes.

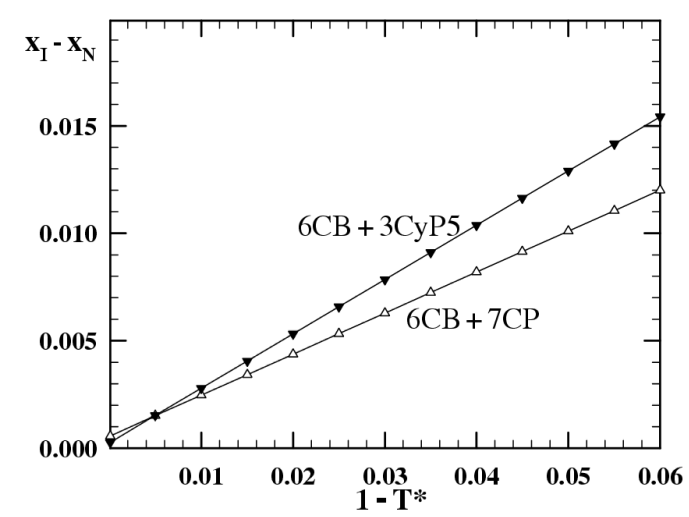

Fig. 3. Concentration extent of the two-phase $\mathrm{I}+\mathrm{N}$ region in $6 \mathrm{CB}+$ non-mesogenic solute mixtures as a function of the reduced temperature (see Eq. (1)).

where $R$ is the gas constant. Figure 3 shows that the linear dependence predicted by Eq. (1) is observed and the slopes of the lines corresponding to two different solutes are close to each other. The entropy jump $\Delta S_{\mathrm{NI}}$, estimated from the data presented in Fig. 3, is equal to $1.785 \mathrm{~J} /(\mathrm{mol} \mathrm{K})$ and $1.538 \mathrm{~J} /(\mathrm{mol} \mathrm{K})$, which gives for the latent heat the values of $466 \mathrm{~J} / \mathrm{mol}$ and $541 \mathrm{~J} / \mathrm{mol}$ for $6 \mathrm{CB}+3 \mathrm{CyP} 5$ and $7 \mathrm{CP}$ mixtures, respectively. The values of $\Delta H$ obtained in our experiment, although very approximate, are situated within the limits of the values obtained with the calorimetric methods for the nematic to the isotropic phase transition of pure $6 \mathrm{CB}$ (from $896 \mathrm{~J} / \mathrm{mol}[10]$ to $293 \mathrm{~J} / \mathrm{mol}[11]$ ).

Figures $4 \mathrm{a}$ and $5 \mathrm{a}$ present temperature dependences of the shear viscosity of the solutions studied. As usual for freely flowing mesogenic liquids, at the isotropic to the nematic phase transition one observes an anomaly in temperature behavior of the viscosity: with decreasing temperature the viscosity strongly decreases, 


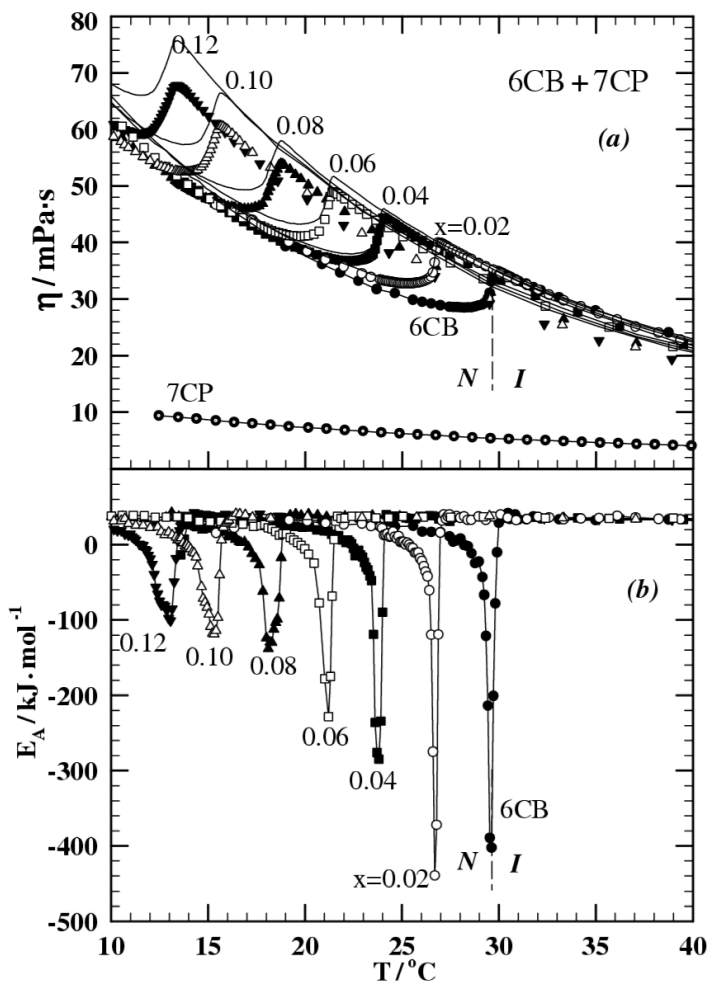

Fig. 4. Temperature dependence of the shear viscosity (a) and the activation energy (b) for freely flowing $6 \mathrm{CB}+7 \mathrm{CP}$ mixtures for different mole fractions of the solute. The solid lines in (a) represent the partial viscosity of $6 \mathrm{CB}$ calculated from Eq. (2).

attains its minimum and next begins to increase, i.e. the viscosity returns to its normal behavior. The results presented clearly show that the temperature extent of the anomalous viscosity behavior is a function of the admixture concentration in solution and reflects the extent of the $\mathrm{I}+\mathrm{N}$ two-phase region in $6 \mathrm{CB}+7 \mathrm{CP}$ and $3 \mathrm{CyP} 5$ solutions, respectively. A quantitative analysis of the measured viscosity $\eta_{\text {meas }}(T)$, leading to some conclusions on the isotropic to the nematic phase transition in $6 \mathrm{CB}$ more and more modified by an increasing concentration of non-mesogenic molecules, must take into account the admixture viscosity temperature dependence $\eta_{\mathrm{adm}}(T)$. The dependences obtained for pure admixtures $7 \mathrm{CP}$ and $3 \mathrm{CyP} 5$ are presented in Figs. $4 \mathrm{a}$ and $5 \mathrm{a}$, respectively. Now, if one assumes that in relatively diluted solutions, as studied here, the viscosity additivity holds:

$$
\eta_{\text {meas }}=(1-x) \eta_{6 \mathrm{CB}}+x \eta_{\mathrm{adm}},
$$

one can calculate from Eq. (2) a "partial" viscosity of $6 \mathrm{CB}\left(\eta_{6 \mathrm{CB}}\right)$ in a mixture of a given mole fraction $x$ of the admixture. The solid lines in Figs. 4a and $5 \mathrm{a}$ are the results of these calculations. The "partial" viscosity of $6 \mathrm{CB}$ can be now presented in a form of the Arrhenius plot ( $\ln \eta_{6 \mathrm{CB}}$ vs. $T^{-1}$ ) and the activation energy $E_{\mathrm{A}}$ 


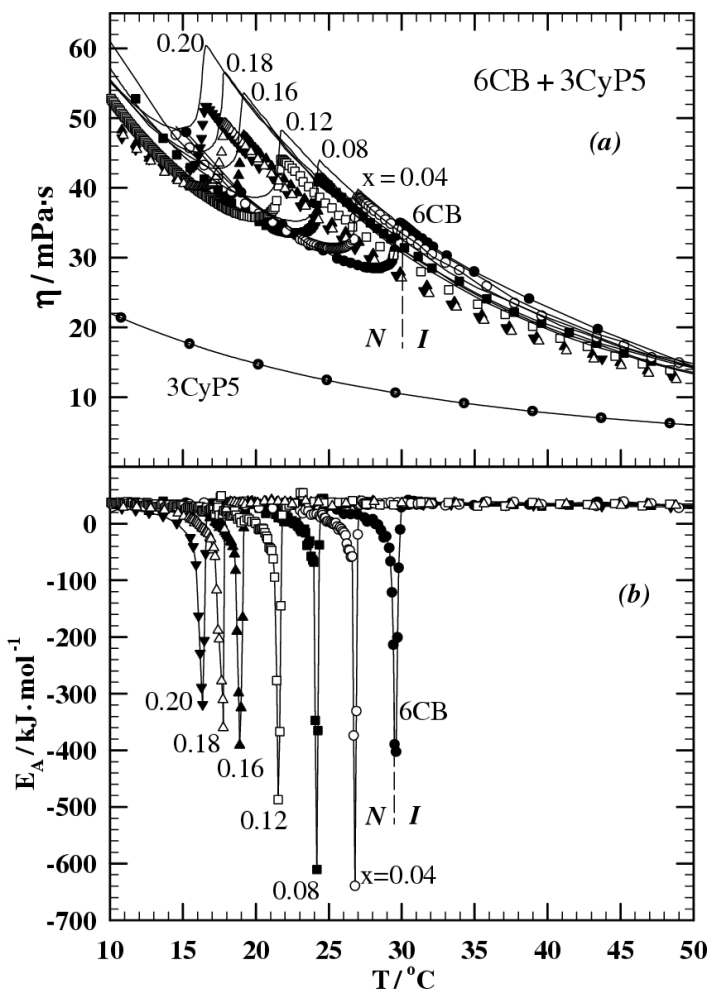

Fig. 5. Temperature dependence of the shear viscosity (a) and the activation energy (b) for freely flowing $6 \mathrm{CB}+3 \mathrm{CyP} 5$ mixtures for different mole fractions of the solute. The solid lines in (a) represent the partial viscosity of 6CB calculated from Eq. (2).

can be calculated according to the following equation:

$$
\ln \eta_{6 \mathrm{CB}}(T)=A+\frac{E_{\mathrm{A}}}{R T} .
$$

Recently, we have shown $[12,13]$ that the most reliable procedure of the determination of the activation energy $E_{\mathrm{A}}$ is based on the differentiation of $\ln \eta_{6 \mathrm{CB}}$ vs. $T^{-1}$ dependence. As results from Eq. (3), the derivative $\partial \ln \eta_{6 \mathrm{CB}} / \partial\left(T^{-1}\right)$ gives $E_{\mathrm{A}}$ directly at a given temperature, allowing one to analyze temperature dependence of the activation energy. Of course, the procedure requires very accurate data for the viscosity determined for an appropriate temperature change step, especially in the vicinity of the phase transition.

Figures $4 \mathrm{~b}$ and $5 \mathrm{~b}$ present the activation energy of the viscous free flow of the solutions of $6 \mathrm{CB}+7 \mathrm{CP}$ and $3 \mathrm{CyP} 5$, respectively, as a function of temperature. An anomalous change of the viscosity observed at the transition from the isotropic liquid to the two-phase $\mathrm{I}+\mathrm{N}$ region in the mixtures studied, manifests itself as a strong decrease in the activation energy up to quite high negative values. As expected, the temperature extent of these peaks strictly corresponds to the 
extent of the two-phase region, where the anomalies in the viscosity behavior are observed. Consequently, in the case of the mixtures of $6 \mathrm{CB}+3 \mathrm{CyP} 5$ (Fig. 5b) the activation energy peaks are exceptionally narrow. Far from the phase transitions, the activation energy for a free flow of the mixtures looks like it is temperature independent, which customarily is taken as the Arrhenius behavior of the system. However, many experiments show that the thermally activated physical processes, in general, do not fulfill the Arrhenius relation (3) with the assumption of the temperature independent activation energy. As we discussed previously $[12,13]$ there are no physical reasons for the assumption. As the basic physical properties of liquids, the number of molecules in a unit volume, first of all, depend essentially on temperature, one may expect some increase in $E_{\mathrm{A}}$ with decreasing temperature, rather than its constant value. Figure 6 seems to be a good illustration of that problem. The activation energy of the viscous flow of the isotropic liquids used in

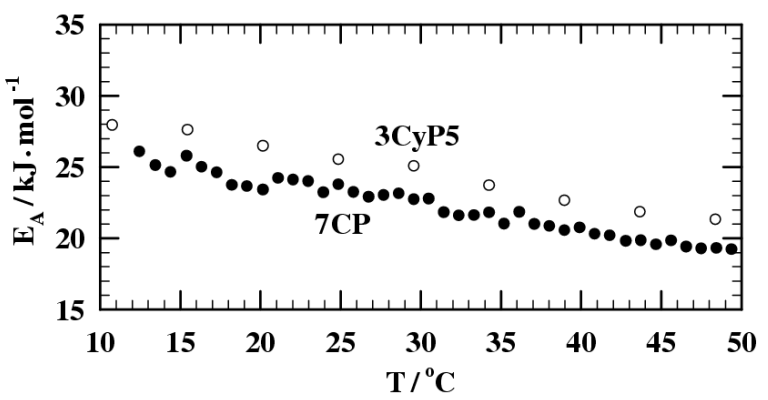

Fig. 6. Temperature dependence of the viscosity activation energy of pure isotropic liquids $7 \mathrm{CP}$ and $3 \mathrm{CyP} 5$.

our experiment as isotropic admixtures shows a weak, but beyond the experimental errors, temperature dependence. The data also show that at a given temperature, the value of $E_{\mathrm{A}}$ which, as a matter of fact, expresses only in a specific way the viscosity temperature dependence, is not sensible to the polarity of the molecules of flowing liquid. The results presented in Fig. 6 show that in spite of the difference in the values of the viscosity of 7CP and 3CyP5 (Figs. 4a and 5a), the temperature behavior of both viscosities are very close to each other.

\section{References}

[1] H.E. Stanley, Introduction to Phase Transitions and Critical Phenomena, Oxford University Press, Oxford 1971.

[2] H.B. Callen, Thermodynamics and an Introduction to Thermostatics, Wiley, New York 1985.

[3] M.A. Anisimov, Critical Phenomena in Liquids and in Liquid Crystals, Gordon and Breach, Reading (MA) 1992.

[4] S. Singh, Phys. Rep. 324, 107 (2000). 
[5] J. Thoen, in: Phase Transitions in Liquid Crystals, Eds. S. Martellucci, A.N. Chester, NATO Advanced Study Institute, Series B: Physics, Plenum, New York 1992, p. 155.

[6] B. Van Roie, J. Leys, K. Denolf, C. Glorieux, G. Pitsi, J. Thoen, Phys. Rev. E 72, 041702 (2005).

[7] P.G. de Gennes, J. Prost, The Physics of Liquid Crystals, 2nd ed., Clarendon Press, Oxford 1993.

[8] D.E. Martire, G.A. Oweimreen, G.I. Ågren, S.G. Ryan, H.T. Peterson, J. Chem. Phys. 64, 1456 (1976).

[9] D.E. Martire, in: The Molecular Physics of Liquid Crystals, Eds. G.R. Luckhurst, G.W. Gray, Academic Press, London 1979, chs. 10 and 11.

[10] E.M. Barrall, R.J. Cox, A. Doelman, N. Clecak, J.A. Logan, A.R. Gregges, Anal. Calorim. 4, 19 (1977).

[11] J. Thoen, Int. J. Modern Phys. B 9, 2157 (1995).

[12] J. Jadżyn, G. Czechowski, C. Legrand, R. Douali, Phys. Rev. E 67, 041705 (2003).

[13] M. Ginovska, H. Kresse, D. Bauman, G. Czechowski, J. Jadżyn, Phys. Rev. E 69, 022701 (2004). 